\title{
LASER SCRIBING AND PRINTING PROCESSES FOR THIN-FILM PV DEVICES
}

\author{
J. Löffler ${ }^{* 1}$, L. Wipliez ${ }^{1}$, M.C.R. Heijna ${ }^{1}$, L.H. Slooff-Hoek ${ }^{1}$, M.A. de Keijzer ${ }^{1}$, J. Bosman ${ }^{1}$, W.J. Soppe ${ }^{1}$, \\ A. Schoonderbeek ${ }^{2}$, J.E.J.M. Rubingh ${ }^{3}$, F. Furthner ${ }^{3}$, P.G.M. Kruijt ${ }^{4}$ \\ *Corresponding author, phone +31 (224) 56 4421, e-mail: loffler@ecn.nl \\ ${ }^{1}$ ECN, Solar Energy, P.O. Box 1, 1755 ZG Petten, The Netherlands. ${ }^{2}$ Laser Zentrum Hannover e.V., Hollerithallee 8, \\ D-30419 Hannover, Germany. ${ }^{3}$ Holst Centre, PO Box 8550, 5605 KN Eindhoven, The Netherlands. ${ }^{4}$ Philips Business \\ Center for OLED lighting, Philipsstraße 8, 52068 Aachen, Germany.
}

\begin{abstract}
Generic processes such as depth-selective laser scribing and the printing of insulating and conductive tracks that can be cured at low temperature, are a key requirement to enable cost efficient production of advanced thin-film PV concepts. In contrast to TCO glass based superstrate device concepts, no standard laser scribing processes and manufacturing tools to achieve fully monolithic series interconnection are presently available for so-called substrate type device concepts that are built on - often opaque and/orflexible - substrates. Three key features of such advanced interconnection processes are presented here: laser scribing, printing and electrical modeling. As vehicle to develop and demonstrate these interconnection processes, a novel roll-to-roll concept for fabrication of thin-film silicon solar cells on steel foil is chosen. We show that laser scribing of amorphous silicon solar cells with a $1064 \mathrm{~nm}$ ps laser leads to a minor loss in efficiency. The screen printing process of low-temperature curing $\mathrm{Ag}$ paste is also proven not to degrade the device performance, and by modeling the module interconnection, we calculated losses below $10 \%$ when going from individual cells to modules. Efforts are underway to realize large area solar cells with printed current collecting grids, and finally a fully series interconnected module by combining laser scribing and printing as cost-effective processing operation.
\end{abstract}

\section{INTRODUCTION}

For thin-film PV concepts based on TCO coated glass superstates, especially when based on amorphous and microcrystalline silicon, standard processes and laser scribing tools are available to achieve monolithically series interconnected modules [1]. Other thin-film PV concepts are based on substrates which are often opaque and also flexible either to enable cost-effective roll-to-roll production, or for applications that require particularly shaped PV laminates, integration into building elements, or even flexibility of the complete PV product. For all (Silicon, CIGS, CdTe, OPV) substrate based thin-film PV technologies, additional requirements have to be met for the interconnection processes: the laser scribing step for the thin-films requires depth selectivity, and printing of insulating and conductive inks may be needed. The curing process for these printing inks has to be done at low temperature, and low sheet resistance and low contact resistance to the front TCO contact and to the rear contact of the devices, which becomes accessible after laser scribing, have to be achieved. Besides, it is crucial that damages to the devices due to the laser scribing, the printing or the curing processes are avoided. When combining the laser patterning and printing steps towards monolithic integration, the layout of the fabricated modules should be optimized for maximum device efficiency, aiming at minimal overall losses from optical shading and ohmic line and contact resistances.

Actually, no standard high-throughput processes for depth-selective laser scribing of thin-film PV devices on (opaque, flexible) substrates or low-temperature printed metallization are readily available.

In this work, the ablation thresholds of the different individual layers constituting thin-film Si solar cells on steel foil/insulating barrier substrates are determined for nine different ns to fs pulsed lasers. The best laser parameters for depth-selective P1, P2 and P3 scribes are tested on small thin-film silicon solar cells. For printing and curing of metal at low substrate temperature, recently more and more materials and techniques have become available. Here the full trajectory from material and process selection and their evaluation till integration into representative devices is presented, yielding remaining issues and (approaches to) their solution. A new finite element model to simulate module losses and optimize metallization patterns will allow more precise calculations than existing models since effects like dark diodes under the metallization, contact resistances in the interconnect, shunt distributions, possible local deterioration of device quality due to printing/laser scribing, etc can also be taken into account.

\section{EXPERIMENTAL}

As vehicle for the process development, a novel concept for low-cost roll-to-roll manufacturing of thin film silicon solar cells and modules [2] is used, which is based on a conducting steel foil substrate coated with an insulating barrier layer. On top of this barrier layer, a sputtered back contact is applied, followed by PECVD of the active silicon layers and the front TCO sputtering. After all solar cell layers are deposited, the three depth-selective laser scribes P1, P2 and P3 are realized in one single step on this full stack, as can be seen in Figure 1. For the P1 scribe, all the layers of the cell have to be ablated including the back contact, while the insulating layer should be unaffected. This scribe will be subsequently filled by an insulating ink. The laser scribe P2 is necessary 
for the actual connection of the front contact of one cell to the back contact of the adjacent cell. P2 should remove all silicon layers, but leave the $\mathrm{ZnO} / \mathrm{Ag}$ back contact unaffected. This scribe will be then filled by an electrically conductive ink.

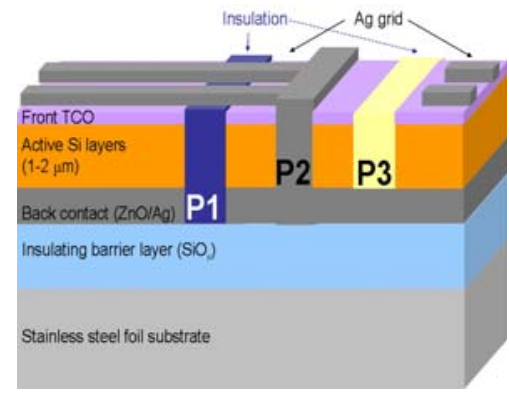

Figure 1. ECN concept for thin-film silicon devices on foil substrate.

In order to evaluate the interaction between laser pulses and a stack of a-Si and $\mu \mathrm{c}-\mathrm{Si}$ layers, a starting point is scribing separated spots on the sample, using different ns to fs pulsed lasers. Microscopy is then used to determine the depth selectivity (P1 and P2/P3) and to assess the quality of the spots, in order to choose the optimum wavelength-pulse duration combination. Spots are subsequently overlapped to scribe lines, aiming at finding best laser parameters in terms of depth selectivity and cleanest scribes. Those parameters are afterwards tested on cells whose I-V curve is measured before and after laser scribing. Screen printing and inkjet printing of commercially available inks have been selected as most promising metallization technologies. For both processes, baseline runs have been executed on glass and ITO coated glass. Besides, current collecting grids have been fabricated on top of small solar cells to evaluate the influence of the printing and curing steps on the cell performance. First estimations on module interconnection losses and optimized patterns have been made based on an existing model. Currently, a more advanced Finite Element Model (FEM) is under development.

\section{RESULTS}

\section{Laser scribing}

The determination of the peak fluence ablation thresholds $\varphi_{\text {th }}$ (in $\mathrm{J} / \mathrm{cm}^{2}$ ) [3] of the different individual layers constituting the device allows us to compare different lasers with ns to fs pulse duration at wavelength of 1064 $\mathrm{nm}$ (near IR), $532 \mathrm{~nm}, 355 \mathrm{~nm}$ and $266 \mathrm{~nm}$ (UV). Figure 2 below illustrates the ablation thresholds for P2 and P1 spots, and areas where the back contact is not visible (in grey) and where the barrier layer is completely removed (in purple) as a function of fluence, for the nine lasers tested on our sample. This figure combined with the optical and confocal microscope studies of the spots allows us to identify the NIR ps laser as one of the most suitable ones for depth-selective laser scribes regarding quality and process window.

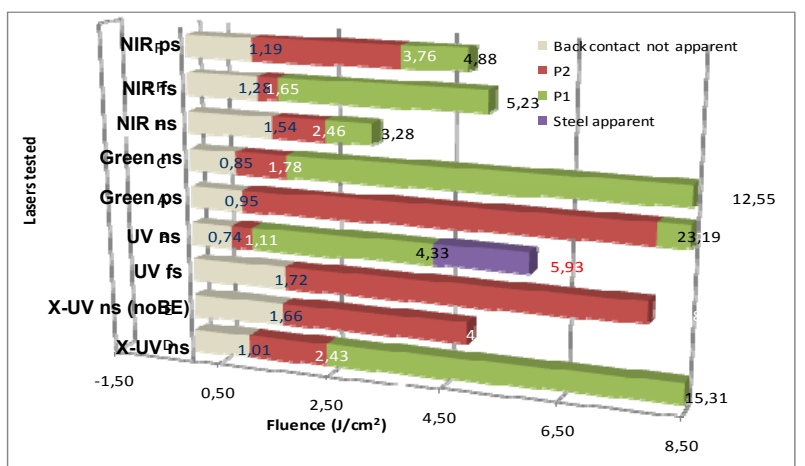

Figure 2. Ablation thresholds and process windows for nine different lasers on the ECN full stack sample.

When overlapping spots with this laser, clean P1 and P2/P3 laser scribes are obtained and tested on functional solar cells whose photoelectrical properties are measured before and after laser scribing.
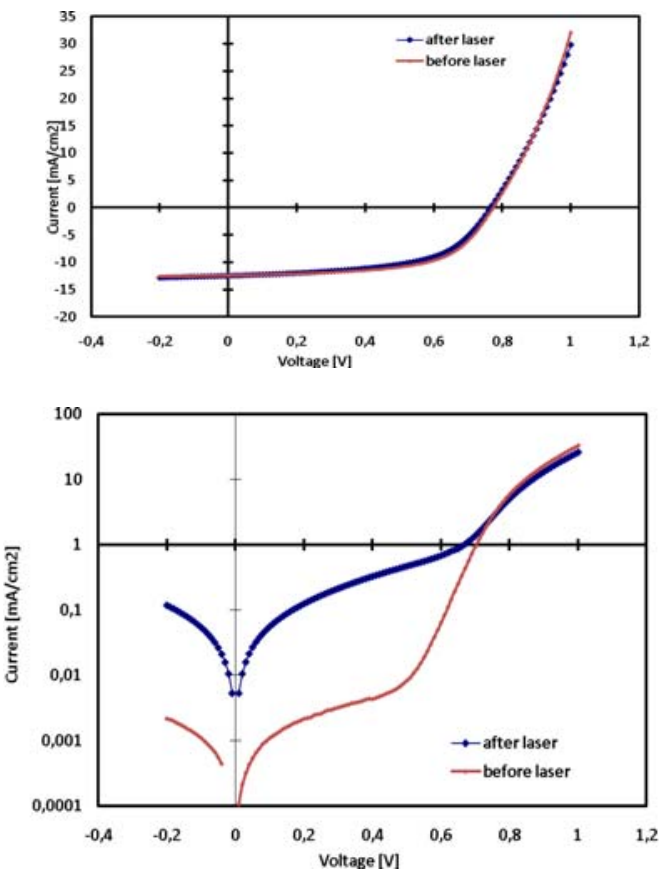

Figure 3. I-V curve under illumination (top) and dark (bottom) before and after laser scribing a P1 line.

The I-V curve under light of this cell (see Figure 3), can almost be overlaid on the I-V measurement before laser scribing, proving that this laser induces minor losses in electrical performance. These I-V curves under light indicate a loss of only $7 \%$ in efficiency decreasing from 5,8 to $5,4 \%$, affecting principally the fill factor. The effect of the laser scribe on the cell behaviour is better visible on measurements in the dark displayed in the bottom graph of Figure 3, where the dark current for a voltage between 0 and $0,7 \mathrm{~V}$ increased significantly, probably due to small shunts coming from the laser step. Efforts are underway to fabricate series connected devices combining these laser 
processes for $\mathrm{P} 1, \mathrm{P} 2$ and $\mathrm{P} 3$ with the printing of insulating and conductive tracks.

\section{Printing}

First baseline runs have been executed for screen printing and inkjet printing. The main results are displayed in Figure 4. The inkjet printed lines are significantly lower $(<<$ $1 \mu \mathrm{m}$ per pass) compared to the screen printed lines $(\sim 10$ $\mu \mathrm{m})$. Consequently, the sheet resistance of the inkjet printed structures is still a factor of more than 10 times higher than for the screen printing baseline, despite the higher bulk conductivity of the inkjet ink compared to the screen printing paste. For the inkjet printed lines it can further be noticed that the actual line width is about 2-3 times larger than the intended width, and that the sheet resistance decreases with increasing line width. Both observations indicate issues with line definition.

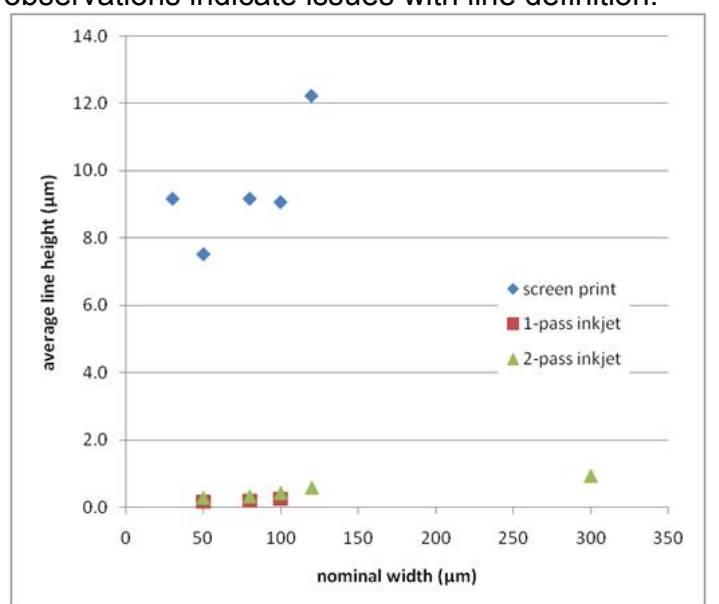

Figure 4. Comparison of screen printed and single and double-pass inkjet-printed metallisation on glass.

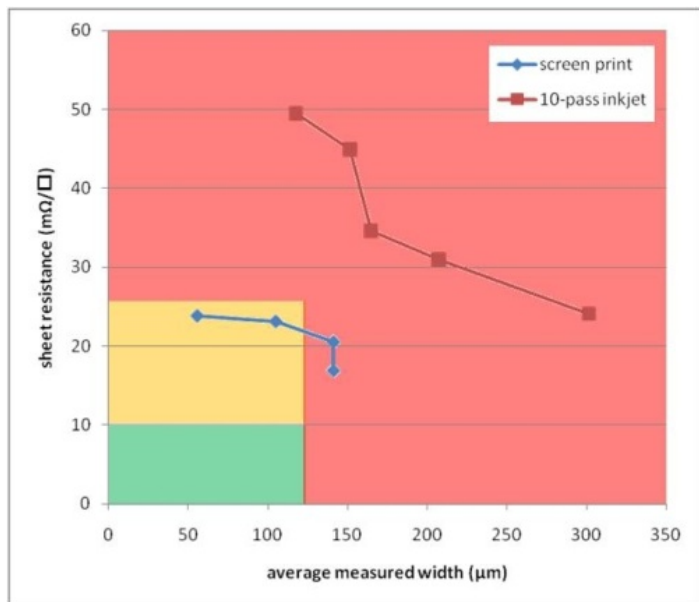

Figure 5. Sheet resistance versus width of screen printed and 10-pass inkjet printed lines. The red, orange and green areas indicate ideal, acceptable and insufficient performance as determined from PV specifications.
In order to overcome the limitations of too low height, the number of passes for inkjet printing has been increased to 10, see Figure 5. This has lead to a significant improvement in sheet resistance, approaching the values for screen printing, however there is still quite a gap. The coloured areas in Figure 5 visualize the challenge to develop inkjet technologies with acceptable (yellow) or even 'ideal' (green) properties for the PV application.

First promising values for the contact resistance between $\mathrm{Ag}$ and ITO of $10 \mathrm{~m} \Omega \mathrm{cm}^{2}$ and $40 \mathrm{~m} \Omega \mathrm{cm}^{2}$ for screen printing and inkjet printing, respectively, have been obtained. As for both values also a relatively large error was determined, work is in progress to improve the method for determining contact resistances.

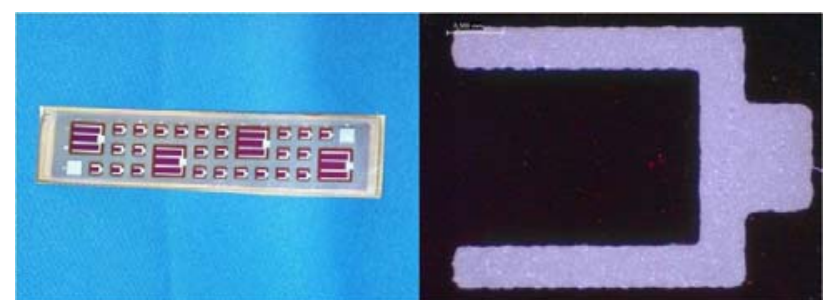

Figure 6. Example of an e-beam evaporated test grid on $4 \times 4 \mathrm{~mm}^{2}$ and $10 \times 10 \mathrm{~mm}^{2}$ cells (left panel) and a microscope image of a screen printed test grid on $4 \times 4$ $\mathrm{mm}^{2}$ cells (right panel).

Screen printed current collecting grids and grids made by e-beam evaporation (see Figure 6) yield comparable results as is shown in

Table 1. The differences in short-circuit current Jsc are within the statistical variations between different cells.

Table 1. Comparison of the best a-Si cell with e-beam evaporated metallisation and with screen printed metallisation.

\begin{tabular}{|l|l|l|l|l|}
\hline Grid & $\begin{array}{l}\mathbf{\eta} \\
{[\%]}\end{array}$ & $\begin{array}{l}\mathbf{V}_{\text {oc }} \\
{[\mathbf{m V}]}\end{array}$ & $\begin{array}{l}\mathbf{J}_{\text {sc }} \\
{\left[\mathbf{m A} / \mathbf{c m}^{2}\right]}\end{array}$ & $\begin{array}{l}\mathbf{F F} \\
{[\%]}\end{array}$ \\
\hline E-beam & 6.0 & 788 & 14.0 & 54.4 \\
\hline $\begin{array}{l}\text { Screen } \\
\text { print }\end{array}$ & 5.8 & 797 & 13.2 & 55.2 \\
\hline
\end{tabular}

In a later experiment, also inkjet printed grids have been tested and yielded comparable results with screen printing, see Table 2.

Table 2. Comparison of a-Si cells with screen printed and inkjet printed metallisation.

\begin{tabular}{|l|l|l|l|l|}
\hline Grid & $\begin{array}{l}\mathbf{\eta} \\
{[\%]}\end{array}$ & $\begin{array}{l}\mathbf{V}_{\text {oc }} \\
{[\mathbf{m V}]}\end{array}$ & $\begin{array}{l}\mathbf{J}_{\mathbf{s c}} \\
{\left[\mathbf{m A} / \mathbf{c m}^{2}\right]}\end{array}$ & $\begin{array}{l}\mathbf{F F} \\
{[\%]}\end{array}$ \\
\hline $\begin{array}{l}\text { Screen } \\
\text { print }\end{array}$ & 5.7 & 797 & 11.6 & 62.1 \\
\hline $\begin{array}{l}\text { Inkjet } \\
\text { print }\end{array}$ & 5.7 & 785 & 11.7 & 61.5 \\
\hline
\end{tabular}

This shows that both printing technologies and the used inks do not harm the flexible thin-film Silicon solar cells. In order to judge about the influence of series and contact 
resistances, larger cells and devices with higher fill factors are necessary, which are currently developed.

In a next step, efforts are underway to fabricate such large solar cells on foil $\left(\sim 10 \times 10 \mathrm{~cm}^{2}\right)$ with low-temperature curing printed current collecting grids, and fully series interconnected modules combining laser scribing and printing.

\section{Integration}

The present version of the PatOpt simulation tool restricts the grid optimisation to $\mathrm{H}$-patterns and only takes into account the front side of the wafer equivalent device. Nevertheless, series integration losses below $10 \%$ could be predicted based on currently achieved metallization results.

A more refined finite elements (FEM) based model is developed to allow for free choice of grid pattern and to include the influences on efficiency losses on module level from back contact resistance and phenomena like dark diode current under grid fingers. Figure 7 gives an example of a first FEM trial run modelling the grid pattern of our standard $1 \mathrm{~cm}^{2}$ test cells.

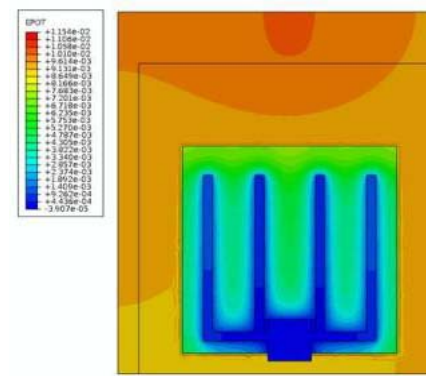

Figure 7. Example of first trial run modelling test grids using a finite elements software showing the potential distribution.

\section{SUMMARY AND CONCLUSIONS}

Generic processes such as depth-selective laser scribing and the printing of insulating and conductive tracks that can be cured at low temperature, are a key requirement to enable cost efficient production of substrate type thin-film $\mathrm{PV}$ concepts which are often built on opaque and even flexible substrates.

As an example, three key features of a novel roll-to-roll concept for fabrication of thin-film silicon solar cells on steel foil are presented: laser scribing, printing and modelling. We show that laser scribing of $4 \times 4 \mathrm{~mm}^{2}$ amorphous silicon solar cells with a $1064 \mathrm{~nm}$ ps laser leads to a minor loss in efficiency. The screen printing process of low-temperature curing $\mathrm{Ag}$ is also proven not to degrade the device performance, and by modelling the module interconnection, we calculated losses below $10 \%$ when going from individual cells to modules. Efforts are underway to realize large area solar cells with printed current collecting grids, and finally a fully series interconnected module by combining laser scribing and printing as cost-effective processing operation.

\section{ACKNOWLEDGEMENTS}

This work has been supported by the European Commission under grant agreement no. 248816 (project LOTUS).

\section{REFERENCES}

[1] T. Witte et al., Proc. $25^{\text {th }}$ EUPVSEC Valencia, Spain (2010).

[2] B.B. van Aken et al., Proc. $35^{\text {th }}$ IEEE PVSC

Philadelphia, PA, USA (2009).

[3] L. Wipliez et al., Proc. $29^{\text {th }}$ ICALEO Anaheim, CA, USA (2010). 